\title{
Alterações nas Características Fisiológicas de Cultivares de Cana-de-Açúcar Submetida À Aplicação de Herbicidas ${ }^{1}$
}

\author{
Changes in the Physiological Characteristics of Sugarcane Cultivars Submitted to Herbicide \\ Application
}

\author{
TORRES, L.G. ${ }^{2}$, FERREIRA, E.A. ${ }^{3}$, ROCHA, P.R.R. ${ }^{4}$, FARIA, A.T. ${ }^{2}$, GONÇALVES, V.A. ${ }^{2}$, GALON, L. ${ }^{5}$, \\ SILVA, A.F. ${ }^{6}$ e SILVA, A.A. ${ }^{7}$
}

\begin{abstract}
RESUMO - Avaliou-se neste trabalho a influência de herbicidas nas características fisiológicas de três cultivares de cana-de-açúcar. O experimento foi conduzido no delineamento em blocos ao acaso, com três repetições, no esquema fatorial $3 \times 8$. O fator $\mathrm{A}$ foi composto por três cultivares de cana-de-açúcar (RB867515, RB855156 e SP80-1816), e o B, pelos herbicidas (tembotrione, MSMA, diuron + hexazinone, sulfentrazone, trifloxysulfuron-sodium, tebuthiuron, clomazone) e uma testemunha sem uso de herbicidas. A taxa transpiratória das plantas do cultivar RB867515 foi afetada negativamente quando foram aplicados os herbicidas sulfentrazone, tebuthiuron e clomazone em comparação à testemunha. Com relação à eficiência do uso da água, não se observaram diferenças entre os cultivares e a aplicação de herbicidas. A condutância estomática dos cultivares RB867515 e SP80-1816 não foi alterada pelos herbicidas aplicados. Apenas o cultivar RB867515 apresentou taxa fotossintética menor quando se aplicou o sulfentrazone. Os herbicidas testados afetam de forma diferenciada as características fisiológicas nos três cultivares avaliados. O cultivar RB867515 foi o que apresentou menor variação na taxa fotossintética na presença dos produtos testados, em relação à testemunha.
\end{abstract}

Palavras-chave: taxa fotossintética, Saccharum spp., seletividade a herbicidas.

\begin{abstract}
This work aimed to evaluate the influence of herbicides on the physiological characteristics of three sugarcane cultivars. The experiment was conducted in a randomized block design with three replications in a factorial design $3 \times 8$. Factor A was constituted of three sugarcane cultivars (RB867515, RB855156, and SP80-1816), and factor B of the herbicides tembotrione, MSMA, diuron + hexazinone, sulfentrazone, trifloxysulfuron-sodium, tebuthiuron, clomazone, and a treatment without herbicide (control). The transpiration rate of cultivar $R B 867515$ plants was negatively affected when sulfentrazone, clomazone, and tebuthiuron were applied, compared to the control. Regarding wateruse efficiency, no differences were observed between the cultivars and the herbicides. Stomatal conductance of cultivars RB867515 and SP80-1816 was not affected by the herbicides. Only cultivar $R B 867515$ presented lower photosynthetic rate when sulfentrazone was applied. The herbicides tested had distinct effects on the physiological characteristics of the three cultivars evaluated. Cultivar RB867515 showed the least variation in photosynthetic rate in the presence of the products tested, as compared to the control.
\end{abstract}

Keywords: photosynthetic rate, Saccharum spp., herbicide selectivity.

1 Recebido para publicação em 18.7.2011 e aprovado em 12.2.2012.

2 Estudante de graduação do curso de Agronomia, Universidade Federal de Viçosa - UFV, 36570-000 Viçosa-MG, $<$ livia.torres@gmail.com>, ${ }^{3} \mathrm{Eng}^{-}$-Agr ${ }^{\circ}$., D.Sc., Bolsista PNPD, Universidade Federal dos Vales do Jequitinhonha e Mucuri - UFVJM, Diamantina-MG; ${ }^{4}$ Eng-o-Agr $^{0}$., D. Sc., Bolsista PNPD, Universidade Federal Rural do Semi-Árido - UFERSA, Mossoró-RN; ${ }^{5}$ Engo-Agr ${ }^{0}$., D.Sc., Professor Adjunto, Universidade Federal da Fronteira Sul - UFFS, Campus Erechim-RS; ${ }^{6}$ Engo-Agr ${ }^{\circ}$., D.Sc., Pesquisador Embrapa Milho e Sorgo - CNPMS, Sete Lagoas-MG; ${ }^{7}$ Engo$^{-}$Agr ${ }^{\circ}$., D. Sc., Professor Associado, DFT/UFV. 


\section{INTRODUÇÃO}

O controle de plantas daninhas em canaviais brasileiros é realizado quase que exclusivamente com o uso de herbicidas, tanto em pré como em pós-emergência. O uso do método químico de controle deve-se à praticidade, à alta eficiência e ao baixo custo do controle, quando comparado aos demais métodos (Christoffoleti et al., 2006). Apesar dessas vantagens, esses produtos podem exercer efeitos diretos e indiretos sobre a cultura, ocasionando intoxicações das plantas (Das et al., 2003; Rizzardi et al., 2003; Galon et al., 2010a), o que pode ter como consequência a redução das taxas de crescimento e desenvolvimento da cana-de-açúcar, com reflexos negativos na produtividade do canavial. Entretanto, alguns efeitos - como alteração na absorção de nutrientes e desregulação dos mecanismos de defesa da cultura pela influência dos fatores abióticos ou bióticos - não são perceptiveis nem amplamente considerados (Rizzardi et al., 2003), sendo relatados por poucos autores (Feng et al., 2005; Galon et al., 2010a).

Os efeitos dos herbicidas sobre a cultura não devem ser determinados apenas verificando-se os sintomas visuais de intoxicação, uma vez que já são conhecidos exemplos de produtos que podem reduzir a produtividade das culturas sem causar efeitos visualmente detectáveis. No entanto, outros herbicidas podem ocasionar injúrias acentuadas, que desaparecem com o desenvolvimento da cultura (Negrisoli et al., 2004). A seletividade de herbicidas à cana-de-açúcar é dependente das características físico-químicas e da dose utilizada, do estádio de desenvolvimento, da suscetibilidade dos genótipos e das condições edafoclimáticas no momento da aplicação. Pesquisas realizadas por Ferreira et al. (2005) e Galon et al. (2010a) demonstram distintas respostas de genótipos de cana-de-açúcar à aplicação de herbicidas, tendo como consequência problemas de intoxicação e, às vezes, redução da produtividade de colmos e da qualidade tecnológica do produto colhido.

Uma das características influenciadas pelo uso de herbicidas é a taxa fotossintética. Vários são os fatores que influenciam a fotossintese das plantas de cana direta ou indiretamente, como deficiência hídrica, estresse térmico e herbicidas utilizados para o controle de plantas daninhas (Loreto \& Bongi, 1989). Além desses fatores, a concentração interna e externa de gases (Kirschbaum \& Pearcey, 1988) e a composição e intensidade da luz (Sharkey \& Raschke, 1981) podem estar associadas a danos causados por herbicidas (Ferreira et al., 2005). A taxa fotossintética está diretamente relacionada à radiação fotossinteticamente ativa (composição da luz), aos fatores de disponibilidade hídrica e às trocas gasosas (Naves-Barbiero et al., 2000), altamente dependentes da abertura estomática, podendo assim ser boa indicadora da resposta dos genótipos à ação dos herbicidas.

Outra característica importante que poderá ser alterada pela ação dos herbicidas é a eficiência do uso da água pelas plantas. Ela é definida como a quantidade de água evapotranspirada por uma cultura para a produção de certa quantidade de matéria seca (Silva et al., 2007). Assim, culturas mais eficientes no uso da água produzem mais matéria seca por grama de água transpirada. A soja, por exemplo, necessita de $750 \mathrm{~g}$ de água por g de matéria seca acumulada, enquanto o arroz irrigado necessita de $4.700 \mathrm{~g}$ (Baptista et al., 2001). Com relação à cana-de-açúcar, no Brasil há poucos estudos que avaliaram o efeito de herbicidas na eficiência do uso da água em genótipos de cana-de-açúcar. Galon et al. (2010b), ao trabalharem com vários genótipos de cana-de-açúcar, constataram que o RB855113 apresentou a menor eficiência de uso da água quando submetido à aplicação de ametryn e do trifloxysulfuron-sodium.

Considerando que há diferenciação dos genótipos de cana-de-açúcar quanto à tolerância a herbicidas e que estes alteram o crescimento e desenvolvimento da cultura, avaliouse neste trabalho a influência de tembotrione, MSMA, diuron + hexazinone, sulfentrazone, trifloxysulfuron-sodium, tebuthiuron e clomazone sobre as características associadas à fisiologia da planta.

\section{MATERIAL E MÉTODOS}

O experimento foi conduzido em delineamento experimental de blocos ao acaso, com três repetições, em esquema fatorial ( $3 \times 8)$. 
$\mathrm{O}$ fator A foi composto pelos cultivares de canade-açúcar (RB867515, RB855156 e SP80-1816), e o B, pelos herbicidas tembotrione $\left(\operatorname{Soberan}^{\circledR}\right.$ $200 \mathrm{~mL} \mathrm{ha}^{-1}+$ Aureo $^{\circledR} 1,0 \mathrm{~L} \mathrm{ha}^{-1}$ ); MSMA (Volcane ${ }^{\circledR} 3,0 \mathrm{~L} \mathrm{ha}^{-1}$ ); diuron + hexazinone (Velpar-K GRDA ${ }^{\circledR} 2,0 \mathrm{~kg} \mathrm{ha}^{-1}$ ); sulfentrazone (Solara $^{\circledR}$ 1,2 L ha-1); trifloxysulfuron-sodium $\left(\right.$ Envoke $^{\circledR} 30,0 \mathrm{~g} \mathrm{ha}^{-1}+$ Extravon $\left.^{\circledR} 0,2 \% \mathrm{v} / \mathrm{v}\right)$; tebuthiuron (Combine $500 \mathrm{SC}^{\circledR} 2,0 \mathrm{~L} \mathrm{ha}^{-1}$ ); clomazone (Gamit ${ }^{\circledR}$ 3,0 $\mathrm{L} \mathrm{ha}^{-1}$ ), e uma testemunha sem aplicação de herbicidas.

As unidades experimentais foram constituídas por vasos plásticos contendo $12 \mathrm{dm}^{3}$ de substrato (solo + fertilizantes). Como substrato utilizou-se um Latossolo VermelhoAmarelo, previamente corrigido e adubado. De acordo com a análise realizada, o solo apresentou as seguintes características: $\mathrm{pH}$ em água de 4,3; $\mathrm{MO}=2,5 \mathrm{dag} \mathrm{kg}^{-1} ; \mathrm{P}=1,5 \mathrm{mg} \mathrm{dm}^{-3}$; $\mathrm{K}=40 \mathrm{mg} \mathrm{dm}^{-3} ; \mathrm{Al}^{3+}=0,5 \mathrm{cmol}_{\mathrm{c}} \mathrm{dm}^{-3} ; \mathrm{Ca}^{2+}$ $=1,3 \mathrm{cmol}_{\mathrm{c}} \mathrm{dm}^{-3} ; \mathrm{Mg}^{2+}=0,2 \mathrm{cmol}_{\mathrm{c}} \mathrm{dm}^{-3} ; \mathrm{CTC}(\mathrm{t})$ $=2,1 \mathrm{cmol}_{\mathrm{c}} \mathrm{dm}^{-3} ; \mathrm{CTC}(\mathrm{T})=6,39 \mathrm{cmol}_{\mathrm{c}} \mathrm{dm}^{-3}$; $\mathrm{H}+\mathrm{Al}=4,79 \mathrm{cmol}_{\mathrm{c}} \mathrm{dm}^{-3} ; \mathrm{SB}=1,6 \mathrm{cmol}_{\mathrm{c}} \mathrm{dm}^{-3}$; $\mathrm{V}=25 \%$; e argila $=38 \%$. Após o preenchimento dos vasos com o solo, fez-se o plantio de duas gemas em cada unidade experimental, dos cultivares de cana-de-açúcar. Aos dez dias após a emergência (DAE) efetuou-se o desbaste, deixando-se uma planta por vaso.

A aplicação dos herbicidas foi feita aos 50 DAE da cana-de-açúcar, tendo esta de quatro a seis folhas completamente expandidas. Para aplicação dos produtos utilizou-se um pulverizador costal pressurizado por gás carbônico, equipado com uma barra contendo uma ponta de pulverização da série TT 110.02, calibrado para aspergir $150 \mathrm{~L} \mathrm{ha}^{-1}$ de calda herbicida. Aos 45 dias após a aplicação dos herbicidas, foram realizadas as avaliações fisiológicas, no terço médio da primeira folha completamente expandida, do perfilho principal das plantas de cana-de-açúcar. Para isso, utilizou-se um analisador de gases no infravermelho (IRGA LCA Pro ${ }^{+}$, em casa de vegetação aberta, permitindo livre circulação do ar. Foram avaliadas a taxa fotossintética $\left(\mu \mathrm{mol} \mathrm{m} \mathrm{m}^{2} \mathrm{~s}^{-1}\right)$, a condutância estomática de vapores de água ( $\left.\mathrm{Gs}-\mathrm{mol} \mathrm{m}^{-1} \mathbf{s}^{-1}\right)$, a taxa de transpiração ( $\mathrm{E} \mathrm{mol} \mathrm{H}_{2} \mathrm{O} \mathrm{m}^{2} \mathrm{~s}^{-1}$ ) e a eficiência do uso da água (EUA - $\mathrm{mol} \mathrm{CO}_{2} \mathrm{~mol} \mathrm{H}_{2} \mathrm{O}^{-1}$ ) - esta última obtida pela relação entre quantidade de $\mathrm{CO}_{2}$ fixado pela fotossintese e quantidade de água transpirada. As avaliações foram realizadas no período das 7 às 10 horas.

Todos os dados foram submetidos à análise de variância pelo teste F; em caso de significância, as médias foram agrupadas segundo o critério de Scott-Knott. Todos os testes foram efetuados a $5 \%$ de probabilidade.

\section{RESULTADOS E DISCUSSÃO}

Houve interação entre herbicidas e cultivares para as variáveis taxa fotossintética, condutância estomática de vapores de água e taxa de transpiração e para a eficiência do uso da água.

O cultivar RB867515, quando pulverizado com os herbicidas sulfentrazone, tebuthiuron e clomazone, apresentou menor taxa transpiratória (E) em comparação com a testemunha sem herbicida (Tabela 1). Ressalta-se que esses herbicidas agem diretamente no aparato fotossintético das plantas em que são aplicados, pois o sulfentrazone tem como mecanismo de ação a inibição da rota metabólica de síntese da enzima protoporfirinogênio oxidase (PROTOX), atuando indiretamente na sintese de clorofila em plantas sensiveis. O tebuthiuron é um inibidor do fotossistema II, agindo na proteína D1, e o clomazone, um inibidor da sintese de carotenóides, compostos responsáveis pela proteção da clorofila contra o excesso de luz (Silva et al., 2007). Dessa forma, é esperado que esses produtos interfiram

Tabela 1 - Taxa transpiratória $\left(\mathrm{E}-\mathrm{mol} \mathrm{H}_{2} \mathrm{O} \mathrm{m}^{-2} \mathrm{~s}^{-1}\right)$ de plantas de cana-de-açúcar, em função de cultivares e herbicidas, aos 45 dias após a aplicação dos produtos. Viçosa-MG, 2010

\begin{tabular}{|l|c|c|c|}
\hline \multirow{2}{*}{\multicolumn{1}{|c|}{ Herbicida }} & \multicolumn{3}{|c|}{ Cultivar } \\
\cline { 2 - 4 } & RB 867515 & RB 855156 & SP 80-1816 \\
\hline Tembotrione & $2,66 \mathrm{Aa}^{\underline{1}}$ & $2,57 \mathrm{Aa}$ & $2,62 \mathrm{Aa}$ \\
\hline MSMA & $2,76 \mathrm{Aa}$ & $2,56 \mathrm{Aa}$ & $2,06 \mathrm{Bb}$ \\
\hline Diuron + hexazinone & $2,87 \mathrm{Aa}$ & $2,54 \mathrm{Aa}$ & $3,05 \mathrm{Aa}$ \\
\hline Sulfentrazone & $2,08 \mathrm{Bb}$ & $2,17 \mathrm{Bb}$ & $2,81 \mathrm{Aa}$ \\
\hline Trifloxysulfuron-sodium & $2,70 \mathrm{Aa}$ & $2,24 \mathrm{Bb}$ & $1,82 \mathrm{Bb}$ \\
\hline Tebuthiuron & $2,24 \mathrm{Bb}$ & $2,03 \mathrm{Bb}$ & $2,83 \mathrm{Aa}$ \\
\hline Clomazone & $2,35 \mathrm{Ab}$ & $2,07 \mathrm{Ab}$ & $2,77 \mathrm{Aa}$ \\
\hline Testemunha & $2,85 \mathrm{Aa}$ & $2,87 \mathrm{Aa}$ & $2,72 \mathrm{Aa}$ \\
\hline \multicolumn{1}{|c|}{$\mathrm{CV}(\%)$} & & 14,20 \\
\hline
\end{tabular}

'Médias seguidas pelas mesmas letras, maiúsculas na linha e minúsculas na coluna, constituem grupos homogêneos, conforme Scott-Knott $(\mathrm{p} \leq 0,05)$. 
de forma indireta na E, por atuarem no aparato fotossintético. A presença dos herbicidas reduz a condutância estomática nas plantas sensíveis e, muitas vezes, em plantas tolerantes. Isso geralmente ocorre pelo fechamento dos estômatos, o qual é influenciado por diversos fatores, como disponibilidade hídrica, luz e energia, poluição e herbicidas usados no controle de plantas daninhas (Ometto et al., 2003).

A aplicação de sulfentrazone, trifloxysulfuron-sodium, tebuthiuron e clomazone sobre o cultivar RB855156 ocasionou redução da E. Entretanto, o trifloxysulfuron-sodium e o MSMA afetaram de forma negativa a $\mathrm{E}$ do cultivar SP80-1816 (Tabela 1). O trifloxysulfuron-sodium é um inibidor da ALS; sua ação na fotossintese é indireta, pois ele atua sobre a produção dos aminoácidos de cadeia ramificada valina, leucina e isoleucina, responsáveis pela produção de proteínas em plantas.

Comparando a influência de cada herbicida sobre os cultivares, verificou-se que o tembotrione e diuron + hexazinone não afetaram a E. O MSMA agiu de forma negativa sobre o SP80-1816 para essa variável em relação aos demais cultivares; de forma contrária, nesse cultivar, foi o que demonstrou menor dano sobre a E quando se usou sulfentrazone ou tebuthiuron. O RB867515

Tabela 2 - Eficiência de uso de água (EUA - $\mathrm{mol} \mathrm{CO}_{2} \mathrm{~mol} \mathrm{H}_{2} \mathrm{O}^{-1}$ ) em plantas de cana-de-açúcar, em função de cultivares e herbicidas, aos 45 dias após a aplicação dos produtos. Viçosa-MG, 2010

\begin{tabular}{|l|c|c|c|}
\hline \multirow{2}{*}{\multicolumn{1}{|c|}{ Herbicida }} & \multicolumn{3}{|c|}{ Cultivar } \\
\cline { 2 - 4 } & RB 867515 & RB 855156 & SP 80-1816 \\
\hline Tembotrione & $8,06 \mathrm{Aa}^{1 /}$ & $9,50 \mathrm{Aa}$ & $6,40 \mathrm{Aa}$ \\
\hline MSMA & $6,36 \mathrm{Aa}$ & $5,65 \mathrm{Aa}$ & $6,29 \mathrm{Aa}$ \\
\hline Diuron + hexazinone & $7,37 \mathrm{Aa}$ & $8,43 \mathrm{Aa}$ & $6,95 \mathrm{Aa}$ \\
\hline Sulfentrazone & $5,10 \mathrm{Aa}$ & $6,88 \mathrm{Aa}$ & $8,69 \mathrm{Aa}$ \\
\hline Trifloxysulfuron-sodium & $7,53 \mathrm{Aa}$ & $7,04 \mathrm{Aa}$ & $7,49 \mathrm{Aa}$ \\
\hline Tebuthiuron & $7,88 \mathrm{Aa}$ & $7,95 \mathrm{Aa}$ & $6,66 \mathrm{Aa}$ \\
\hline Clomazone & $7,28 \mathrm{Aa}$ & $6,14 \mathrm{Aa}$ & $6,64 \mathrm{Aa}$ \\
\hline Testemunha & $10,12 \mathrm{Aa}$ & $8,41 \mathrm{Aa}$ & $9,56 \mathrm{Aa}$ \\
\hline \multicolumn{1}{|c|}{$\mathrm{CV}(\%)$} & & 22,62 \\
\hline
\end{tabular}

1/ Médias seguidas pelas mesmas letras, maiúsculas na linha e minúsculas na coluna, constituem grupos homogêneos, conforme Scott-Knott $(\mathrm{p} \leq 0,05)$ apresentou a maior E, comparativamente aos demais, na presença do trifloxysulfuronsodium (Tabela 1).

Com relação à eficiência do uso da água (EUA), não foram observadas diferenças dentro de cada cultivar e dos herbicidas (Tabela 2). A eficiência do uso da água é caracterizada como a quantidade de água transpirada por uma cultura para a produção de certa quantidade de matéria seca (Silva et al., 2007). Assim, culturas mais eficientes no uso da água podem produzir quantidade maior de matéria seca por grama de água transpirada. O uso mais eficiente da água está diretamente relacionado ao tempo de abertura estomática, pois, enquanto a planta absorve $\mathrm{CO}_{2}$ para a fotossíntese, a água é perdida por transpiração, com intensidade variável, dependendo do gradiente de potencial entre a superficie foliar e a atmosfera, seguindo uma corrente de potenciais hídricos (Concenço et al., 2007).

No tocante à taxa fotossintética (A), observou-se que apenas o herbicida sulfentrazone provocou redução no RB867515; os demais tratamentos não diferiram da testemunha (Tabela 3). Ferreira et al. (2005) avaliaram cultivares de cana-de-açúcar e constataram que o RB867515 foi o menos afetado pela aplicação da mistura formulada comercialmente de ametryn + trifloxysulfuron-sodium, em casa de vegetação.

Tabela 3 - Taxa fotossintética $\left(\mathrm{A}-\mu \mathrm{mol} \mathrm{m}{ }^{2} \mathrm{~s}^{-1}\right)$ de plantas de cana-de-açúcar, em função de cultivares e herbicidas, aos 45 dias após a aplicação dos produtos. Viçosa-MG, 2010

\begin{tabular}{|l|c|c|c|}
\hline \multirow{2}{*}{\multicolumn{1}{|c|}{ Herbicida }} & \multicolumn{3}{|c|}{ Cultivar } \\
\cline { 2 - 4 } & RB 867515 & RB 855156 & SP $80-1816$ \\
\hline Tembotrione & $22,10 \mathrm{Aa}^{1 /}$ & $24,48 \mathrm{Aa}$ & $16,45 \mathrm{Ab}$ \\
\hline MSMA & $17,54 \mathrm{Aa}$ & $14,51 \mathrm{Ab}$ & $13,75 \mathrm{Ab}$ \\
\hline Diuron + hexazinone & $21,15 \mathrm{Aa}$ & $21,17 \mathrm{Aa}$ & $21,22 \mathrm{Aa}$ \\
\hline Sulfentrazone & $10,56 \mathrm{Ba}$ & $15,59 \mathrm{Bb}$ & $24,94 \mathrm{Aa}$ \\
\hline Trifloxysulfuron-sodium & $20,53 \mathrm{Aa}$ & $15,85 \mathrm{Ab}$ & $13,90 \mathrm{Ab}$ \\
\hline Tebuthiuron & $17,86 \mathrm{Aa}$ & $16,25 \mathrm{Ab}$ & $18,64 \mathrm{Ab}$ \\
\hline Clomazone & $16,89 \mathrm{Aa}$ & $12,96 \mathrm{Ab}$ & $18,45 \mathrm{Ab}$ \\
\hline Testemunha & $28,44 \mathrm{Aa}$ & $24,07 \mathrm{Aa}$ & $26,03 \mathrm{Aa}$ \\
\hline \multicolumn{3}{|c|}{ CV (\%) } & \multicolumn{3}{|c}{27,78} \\
\hline
\end{tabular}

1/ Médias seguidas pelas mesmas letras, maiúsculas na linha e minúsculas na coluna, constituem grupos homogêneos, conforme Scott-Knott $(\mathrm{p} \leq 0,05)$ 
O sulfentrazone causou redução da fotossintese devido à menor sintese de clorofilas estas são proteínas que apresentam função vital, captando a energia luminosa, que é convertida em poder redutor para o processo de fixação e assimilação do $\mathrm{CO}_{2}$ no ciclo de Calvin (Carretero, 2008). Além disso, as clorofilas podem sofrer os danos causados pelas espécies reativas de oxigênio (ROS) (Gan, 2007), reduzindo ainda mais a atividade fotossintética. À medida que aumenta o estresse oxidativo em função do tempo de exposição à luz, os tilacoides são danificados e perdem sua capacidade de realizar fotossintese, devido a danos na maquinaria fotossintética (Tripathy et al., 2007).

$\mathrm{O}$ influxo de $\mathrm{CO}_{2}$ pode ser comprometido devido à atuação dos herbicidas inibidores da enzima PROTOX, sendo um dos fatores a formação de óxido nítrico por meio das ROS. Esse óxido estimula a síntese e atividade do ácido abscísico (ABA) - hormônio que atua regulando o fechamento estomático (Mata \& Lamattina, 2001). Também pode ocorrer o fechamento estomático pela ação das ROS, favorecendo o acúmulo de cálcio no citossol (Taiz \& Zeiger, 2009), ou ainda devido à peroxidação das membranas celulares das células adjacentes aos estômatos.

No RB855156, os herbicidas MSMA, sulfentrazone, trifloxysulfuron-sodium, tebuthiuron e clomazone provocaram redução na A (Tabela 3). Já no SP80-1816, o diuron + hexazinone e o sulfentrazone não afetaram a variável estudada. É importante destacar que na mistura diuronthexazinone, apesar de ambos os herbicidas pertencerem ao grupo dos inibidores do fotossistema II, não foi observada diferença na A para os três cultivares em relação à testemunha (Tabela 3). Galon et al. (2010a), ao trabalharem com seis cultivares de cana-de-açúcar, verificaram que, para o ametryn, o cultivar RB855113 apresentou A similar à da testemunha, e os demais cultivares demonstraram decréscimo dessa característica em estudo.

Ao avaliar os cultivares dentro de cada herbicida, verificou-se que não houve diferenças entre eles para todos os produtos testados, exceto o sulfentrazone, em que o SP80-1816 demonstrou maior A (Tabela 3). Galon et al. (2010a) observaram que a taxa fotossintética nas plantas tratadas com trifloxysulfuronsodium em seis cultivares de cana-de-açúcar foi similar à da respectiva testemunha sem aplicação de herbicidas, para todos os genótipos avaliados no referido estudo.

A fotossintese e, consequentemente, a respiração dependem de constante fluxo de $\mathrm{CO}_{2}$ e $\mathrm{O}_{2}$ entrando e saindo da célula; esse fluxo livre se dá em função da concentração desses elementos nos espaços intercelulares dependentes da abertura estomática - controladora majoritária do fluxo de gases (Messinger et al., 2006). Esta, por sua vez, é em grande parte controlada pela turgescência tanto das células-guarda (que controlam a abertura dos estômatos) como das células epidérmicas dos estômatos (Humble \& Hsiao, 1970). Desse modo, qualquer efeito, causado pelos herbicidas avaliados, que leve à menor absorção ou à translocação de água pode alterar primeiramente a condutância estomática e/ou mesofílica, reduzindo a taxa fotossintética. Os herbicidas estudados neste trabalho, a excessão do MSMA e do trifloxysulfuron-sodium, afetaram diretamente a taxa fotossintética dos cultivares de cana-de-açúcar. Esses compostos agem no cloroplasto e inibem o transporte de elétrons ou a sintese de clorofila ou, ainda interferem na sintese de pigmentos responsáveis pela proteção do aparato fotossintético.

Nos cultivares RB867515 e SP80-1816 não se observaram diferenças entre os herbicidas para a condutância estomática (Gs); contudo, para o RB855156, a Gs apresentou redução na presença de todos os herbicidas testados (Tabela 4). Ao avaliar a Gs dos cultivares dentro de cada herbicida, não foi observada diferença entre elas. No entanto, na ausência de qualquer herbicida, o cultivar RB855156 apresentou maior Gs (Tabela 4). A redução da condutância estomática foi observada em soja e Portulaca oleracea seis horas após a aplicação do lactofen - inibidor da enzima PROTOX (Wichert \& Talbert, 1993). Esse produto pode promover o fechamento estomático devido aos processos oxidativos e aumento da concentração de óxido nítrico, como já comentado, que é um sinalizador de ABA, hormônio que regula o fechamento estomático (Mata \& Lamattina, 2001). Essa atuação do óxido nítrico tem sido observada em plantas de soja após a aplicação de lactofen (Carretero, 2008). 
Tabela 4 - Condutância estomática de vapores de água (Gs - mol m $\mathrm{m}^{-1} \mathrm{~s}^{-1}$ ) em plantas de cana-de-açúcar, em função de cultivares e herbicidas, aos 45 dias após a aplicação dos produtos. Viçosa-MG, 2010

\begin{tabular}{|l|c|c|c|}
\hline \multirow{2}{*}{ Herbicida } & \multicolumn{3}{|c|}{ Cultivar } \\
\cline { 2 - 4 } & RB 867515 & RB 855156 & SP 80-1816 \\
\hline Tembotrione & $0,67 \mathrm{Aa}^{1 /}$ & $0,46 \mathrm{Ab}$ & $0,48 \mathrm{Aa}$ \\
\hline MSMA & $0,60 \mathrm{Aa}$ & $0,46 \mathrm{Ab}$ & $0,28 \mathrm{Aa}$ \\
\hline Diuron + hexazinone & $0,74 \mathrm{Aa}$ & $0,44 \mathrm{Ab}$ & $0,70 \mathrm{Aa}$ \\
\hline Sulfentrazone & $0,27 \mathrm{Aa}$ & $0,34 \mathrm{Ab}$ & $0,59 \mathrm{Aa}$ \\
\hline Trifloxysulfuron-sodium & $0,52 \mathrm{Aa}$ & $0,31 \mathrm{Ab}$ & $0,20 \mathrm{Aa}$ \\
\hline Tebuthiuron & $0,33 \mathrm{Aa}$ & $0,28 \mathrm{Ab}$ & $0,56 \mathrm{Aa}$ \\
\hline Clomazone & $0,36 \mathrm{Aa}$ & $0,27 \mathrm{Ab}$ & $0,57 \mathrm{Aa}$ \\
\hline Testemunha & $0,61 \mathrm{Ba}$ & $1,00 \mathrm{Aa}$ & $0,47 \mathrm{Ba}$ \\
\hline \multicolumn{1}{|c|}{$\mathrm{CV}$ (\%) } & & 46,9 \\
\hline
\end{tabular}

1/ Médias seguidas pelas mesmas letras, maiúsculas na linha e minúsculas na coluna, constituem grupos homogêneos, conforme Scott-Knott $(\mathrm{p} \leq 0,05)$.

A condutância foliar é composta em pequena parte pela condutância cuticular da epiderme e, quando os estômatos estão abertos, pela Gs, que é controlada pelas célulasguarda dos estômatos. Assim, a Gs é proporcional ao número e tamanho dos estômatos e diâmetro da abertura dessa estrutura; essa variável depende também de outros fatores, sobretudo os relacionados ao ambiente onde a planta se desenvolve (Brodribb \& Holbrook, 2003). A água é perdida por transpiração apenas quando os estômatos estão abertos. O controle da abertura e o fechamento dos estômatos dependem de uma série de fatores, como radiação solar, nível de $\mathrm{CO}_{2}$ no mesofilo, umidade relativa (déficit de pressão de vapor do ar), potencial hídrico e outros de menor magnitude, como vento, substâncias de crescimento e ritmos endógenos próprios de cada espécie.

De acordo com os resultados, os herbicidas testados alteram de forma diferenciada as características fisiológicas nos três cultivares avaliados. De forma geral, o cultivar RB867515 foi o que apresentou menor variação na taxa fossintética na presença dos produtos testados, em relação à testemunha. Os cultivares SP801816 e RB855156 apresentaram menores taxas fotossintéticas para a maioria do produtos avaliados. Ocorreu redução da condutância estomática do cultivar RB855156 após a aplicação de todos os herbicidas.

\section{AGRADECIMENTOS}

À Fundação de Apoio à Pesquisa de Minas Gerais (FAPEMIG), à Coordenação de Aperfeiçoamento de Pessoal de Nivel Superior (CAPES) e ao Conselho Nacional de Desenvolvimento Científico e Tecnológico (CNPq), pelas bolsas e pelo apoio concedido.

\section{LITERATURA CITADA}

BAPTISTA, J. M. et al. Programa nacional para o uso eficiente da água. Lisboa: Instituto Superior de Agronomia, 2001. 212 p.

BRODRIBB, T. J.; HOLBROOK, N. M. Stomatal closure during leaf dehydration, correlation with other leaf physiological traits. Plant Physiol., v. 132, n. 4, p. $2166-2173,2003$.

CARRETERO, D. M. Efeitos da inibição da protoporfirinogênio IX oxidase sobre as trocas gasosas e fluorescência da clorofila a em plantas de soja (Glycine $\max$ L. Merrill). 2008. 57 f. Dissertação (Mestrado em Fisiologia Vegetal) - Universidade Federal de Viçosa, Viçosa, MG, 2008 .

CHRISTOFFOLETI, P. J. et al. Carfentrazone-ethyl aplicado em pós-emergência para o controle de Ipomoea spp. e Commelina benghalensis na cultura da cana-de-açúcar. Planta Daninha, v. 24, n. 1, p. 83-90, 2006.

CONCENÇO, G. et al. Uso da água em biótipos de azevém (Lolium multiflorum) em condição de competição.

Planta Daninha, v. 25, n. 3, p. 449-455, 2007.

DAS, A. C.; DEBNATH, A.; MUKHERJEE, D. Effect of the herbicides oxadiazon and oxyfluorfen on phosphates solubilizing microorganisms and their persistence in rice fields. Chemosphere, v. 53, n. 5, p. 217-221, 2003.

FENG, P. C. C. et al. Glyphosate inhibits rust diseases in glyphosate-resistant wheat and soybean. Proc. Nat. Acad. Sci., v. 102, n. 48, p. 17290-17295, 2005.

FERREIRA, E. A. et al. Sensibilidade de cultivares de canade-açúcar à mistura trifloxysulfuron-sodium + ametryn Planta Daninha, v. 23, n. 1, p. 93-99, 2005

GALON, L. et al. Influência de herbicidas na atividade fotossintética de genótipos de cana-de-açúcar. Planta Daninha, v. 28, n. 3, p. 591-597, 2010a.

GALON, L. et al. Eficiência do uso da água em genótipos de cana-de-açúcar submetidos a aplicação de herbicidas.

Planta Daninha, v. 28, n. 4, p. 777-784, 2010b.

GAN, S. Senescende processes in plants. Ames: Blackwell Publishing, 2007. p. 322. 
HUMBLE, G. D.; HSIAO, T. C. Light-dependent influx and efflux of potassium of guard cells during stomatal opening and closing. Plant Physiol., v. 46, n. 3, p. 483-487, 1970

KIRSCHBAUM, M. U.; PEARCY, R. W. Gas exchange analysis of the fast phase of photosynthetic induction in Alocasia macrorrhiza. Plant Physiol., v. 87, n. 4, p. 818-821, 1988.

LORETO, F.; BONGI, G. Combined low temperature-high light effects on gas exchange properties of jojoba leaves. Plant Physiol., v. 91, n. 4, p. 1580-1585, 1989.

MATA, C. G.; LAMATTINA, L. Nitric oxide induces estomatal closure and enhances the adaptive plant responses against drought stress. Plant physiol., v. 126, n. 3, p. 1196-1204, 2001

MESSINGER, S. M. et al. Evidence for involvement of photosynthetic processes in the stomatal response to $\mathrm{CO}_{2}$. Plant Physiol., v. 140, n. 2, p. 771-778, 2006.

NAVES-BARBIERO, C. C. et al. Fluxo de seiva e condutância estomática de duas espécies lenhosas sempre-verdes no campo sujo e cerradão. R. Bras. Fisiol. Veg., v. 12, n. 2, p. 119-134, 2000.

NEGRISOLI, E. et al. Seletividade de herbicidas aplicados em pré-emergência na cultura da cana-de-açúcar tratada com nematicidas. Planta Daninha, v. 22, n. 4, p. 567-575, 2004.
OMETTO J. P. H. B. et al. Variação temporal do isótopo estável do carbono em material arbóreo em florestas da região Amazônica. In: CONGRESSO BRASILEIRO DE ECOLOGIA, 4., 2003, Fortaleza. Anais... Rio Claro: Sociedade de Ecologia do Brasil, 2003.

RIZZARDI, M. A. et al. Ação de herbicidas sobre mecanismos de defesa das plantas aos patógenos. Ci. Rural, v. 33, n. 5 , p. $957-965,2003$.

SHARKEY, T. D.; RASCHKE, K. Effect of light quality on stomatal opening in leaves of Xanthium strumarium L. Plant Physiol., v. 68, n. 5, p. 1170-1174, 1981.

SILVA, A. A. et al. Competição entre plantas daninhas e culturas. In: SILVA, A. A.; SILVA, J. F. (Ed.). Tópicos em manejo de plantas daninhas. Viçosa, $\mathrm{MG}$ : Sociedade Brasileira de Ciência do Solo, 2007. p. 17-61.

TAIZ, L.; ZEIGER, E. Fisiologia vegetal. Porto Alegre: Artmed, 2009. p. 449-484.

TRIPATHY, B. C. et al. Impairment of the photosinthetic apparatus by oxidative stress induced by photosensitization reaction of protoporphyrin IX. Biochim. Biophys. Acta, v. 1767, n. 6 , p. $860-868,2007$.

WICHERT, R. A.; TALBERT, R. E. Soybean (Glycine max L.) response to lactofen. Weed Sci., v. 41, n. 1, p. 23-27, 1993. 